\title{
Modelling natural electromagnetic interference in man-made conductors for space weather applications
}

\author{
Larisa Trichtchenko \\ Natural Resources Canada, Ottawa, Canada \\ Correspondence to: Larisa Trichtchenko (larisa.trichtchenko@canada.ca)
}

Received: 12 February 2016 - Revised: 23 March 2016 - Accepted: 4 April 2016 - Published: 14 April 2016

\begin{abstract}
Power transmission lines above the ground, cables and pipelines in the ground and under the sea, and in general all man-made long grounded conductors are exposed to the variations of the natural electromagnetic field. The resulting currents in the networks (commonly named geomagnetically induced currents, GIC), are produced by the conductive and/or inductive coupling and can compromise or even disrupt system operations and, in extreme cases, cause power blackouts, railway signalling mis-operation, or interfere with pipeline corrosion protection systems. To properly model the GIC in order to mitigate their impacts it is necessary to know the frequency dependence of the response of these systems to the geomagnetic variations which naturally span a wide frequency range. For that, the general equations of the electromagnetic induction in a multi-layered infinitely long cylinder (representing cable, power line wire, rail or pipeline) embedded in uniform media have been solved utilising methods widely used in geophysics. The derived electromagnetic fields and currents include the effects of the electromagnetic properties of each layer and of the different types of the surrounding media. This exact solution then has been used to examine the electromagnetic response of particular samples of long conducting structures to the external electromagnetic wave for a wide range of frequencies. Because the exact solution has a rather complicated structure, simple approximate analytical formulas have been proposed, analysed and compared with the results from the exact model. These approximate formulas show good coincidence in the frequency range spanning from geomagnetic storms (less than $\mathrm{mHz}$ ) to pulsations $(\mathrm{mHz}$ to $\mathrm{Hz})$ to atmospherics $(\mathrm{kHz})$ and above, and can be recommended for use in space weather applications.
\end{abstract}

Keywords. Geomagnetism and palaeomagnetism (geomagnetic induction)

\section{Introduction}

Large-scale technological systems using long conductors are exposed to different types of natural electromagnetic variations, from geomagnetic disturbances resulting from solar sources through their impacts on the magnetosphere and ionosphere, to atmospheric sources (lightning), overall covering the frequencies from $0.1 \mathrm{mHz}$ to $1 \mathrm{~Hz}$ (periods from days to seconds) up to $1 \mathrm{kHz}$ respectively (Campbell, 1997).

These natural electromagnetic variations produce electric currents which interfere with ground networks, such as phone cables, power lines and pipelines (Lanzerotti and Gregori, 1986). In power systems they can lead to saturation of power transformers. This creates a distorted AC waveform with high harmonic content that causes mis-operation of protective relays. In severe cases power blackouts have occurred (Boteler et al., 1998). In pipelines, the natural electromagnetic variations can compromise the operations of corrosion protection system (cathodic protection) and even create the possibility for enhanced corrosion to occur (Gummow, 2002). Interference with operations of railway signalling systems has also been reported (Ptitsyna et al., 2008; Wik et al., 2009).

To protect the infrastructure from these hazardous impacts, special mitigation procedures are required, which, in turn, need the detailed estimations of the geomagnetically induced currents (GIC) produced by the electromagnetic interference.

Calculations of the GIC in different man-made conductive structures are usually based on the two-step approach (see, for example, Pirjola, 2002). The first step is defined as "geophysical", providing the geoelectric field values (i.e. the electric field in the ground) in the absence of the network, and the second step is defined as "engineering", i.e. calculating electric currents in any component of the network from its electrical parameters and the ambient geoelec- 
tric field. The geoelectric field values are obtained with the help of numerical models, which in general are a conversion of the geomagnetic data recorded at geomagnetic observatories close to the network under investigation, into the geoelectric field values by use of different models for the earth transfer function (surface impedance). Although magnetotelluric measurements can provide direct data on the geoelectric field, these data cover only time periods and locations of the specific field campaigns, and, therefore, in most cases are not directly applied to calculate the GIC at different times in specific networks.

Two assumptions are normally used, one is that the conductor (network) does not change the ambient electric field and another is that the conductors in the network can be represented by their DC resistances only and, therefore, there is no frequency dependency in the "engineering" part of the modelling.

In this paper the effects of frequency of the natural variations on the electromagnetic field in the presence of the manmade conductors (including inside the conductors) is discussed in detail and the area of applicability of the above two assumptions is identified. Earlier estimations done in studies for pipelines (Campbell, 1978; Viljanen, 1989; Trichtchenko and Boteler, 2001) show that the commonly used DC approach should not give large errors for lower frequencies, i.e. corresponding to periods of $1 \mathrm{~min}$ and above. For power lines and other affected conductors such estimations have not been done yet. To test these assumptions would be important also because it has been pointed out recently that the power grids are susceptible to a higher frequency of geomagnetic variations (Kappenman, 2005). These higher frequencies of up to $1 \mathrm{~Hz}$ can already be included in modelling, because the reliable measurements of the magnetic field at $1 \mathrm{~Hz}$ are now available (for example, see the website at http://intermagnet. org/publication-software/technicalsoft-eng.php).

Thus, there is a need and possibility to include the higher frequencies (up to $1 \mathrm{~Hz}$ ) into GIC modelling, but the assumptions which normally go into modelling should be verified and refined.

In order to rigorously examine the effect of electromagnetic interference on different layered infinitely long cylindrical structures for a continuous spectrum of frequencies and for different types of surrounding media, an analytical model has been developed from first principles. This model provides an "exact" solution that allows numerical examination of the behaviour of the electric fields and currents inside the multiple layers of infinitely long conductive structures embedded in the uniform surrounding media with different electromagnetic properties.

For practical applications in network modelling the use of the exact solution would be rather complicated and, therefore, simple approximate formulas were proposed and verified by their comparison with the exact solution.

The paper is structured as follows. Section 2 describes the theoretical approach and gives the derivation of basic formu-

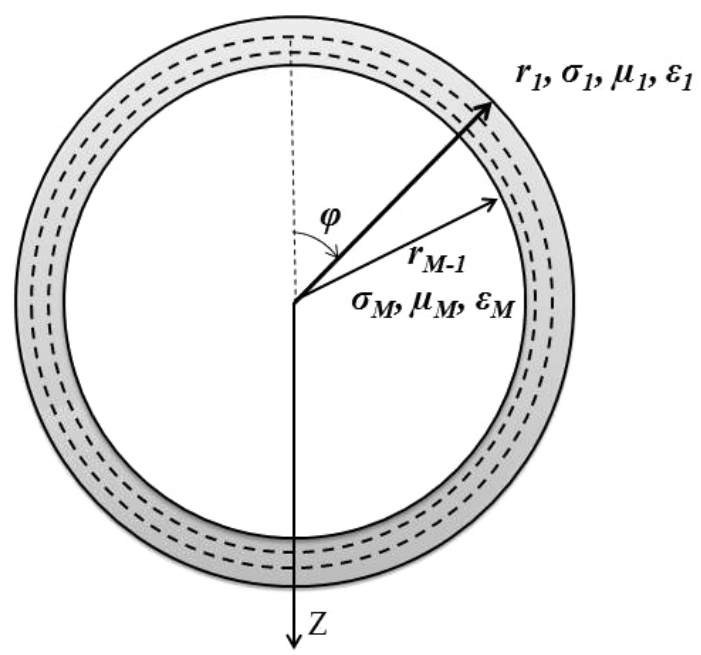

Figure 1. Schematics of the cylinder layers and coordinate system. The axis of the cylinder is coincided with $x$ axis, propagation of the plane wave is along $z$ axis, angle $\varphi$ is clockwise as shown, the external radius is $r_{1}$, the internal is $r_{M-1}$, intermediate layers from $r_{2}$ to $r_{M-2}$ (dashed circles). The corresponding electromagnetic characteristics are indexed as 1 for outside media, $(2, \ldots, M-1)$ for intermediate and $M$ for the innermost.

las which form the "exact" solution. Section 3 presents the electromagnetic and dimensional characteristics of the modelled conductive structures and the surrounding media used as the inputs into the modelling. An illustrative example for interaction of a plane wave with particular single frequency and the most complex layered structure (pipeline) among these discussed is presented in Sect. 4. Section 5 describes the frequency dependence of the results of exact solutions for electromagnetic interference in terms of the electric field inside the layers of conductors. Evaluations of the approximate formulas for effective impedance (composed of both a real part, i.e. Ohmic resistance, and an imaginary part), which can be used to obtain the electric current inside the different conductors from the ambient geoelectric field are presented and discussed in Sect. 6, which is then followed by Conclusions.

\section{Theoretical approach}

This section gives the general expressions which fully describe the electromagnetic response of a multi-layered infinitely long cylindrical conductor placed in uniform lowconducting medium to the variations of the incident electromagnetic field.

The approach presented in Kaufman and Keller (1981) has been chosen as a mathematical basis for this model. The nondisturbed (ambient) geoelectric field in the absence of the cylinder is assumed to be in the form of a plane wave incident to the cylinder. The Cartesian coordinate system with origin 
at the centre of cylindrical conductor is used with horizontal components; $x$ is parallel to the cylinder axis, $y$ is perpendicular to the axis and $z$ is "downwards" (in the propagation direction).

The described domain is shown in Fig. 1 and has the following indexing system: the outside uniform media with its electromagnetic characteristics has index 1 , the cylinder layers, each with its corresponding electromagnetic characteristic, are indexed as $(2, \ldots, M-1, M)$ with their inner radii as $(2, \ldots, M-1)$. The main steps of the derivation are given below for the case of the electric field vector parallel to the cylinder's axis. This orientation is chosen because only the electric field in the direction of the conductors in the network is taken into account in the GIC models. More detailed derivations for the case of a pipeline as the multi-layered cylinder with two different orientations of the incident plane wave are presented in Trichtchenko and Boteler (2001).

For the quasi-stationary electromagnetic field with $E$ parallel to the axis of the infinitely long cylinder, the electric field in any layer satisfies the equation

$\nabla^{2} E-k^{2} E=0$

where $k$ is a propagation constant given by

$k=\sqrt{i \mu \omega(\sigma+i \omega \varepsilon)}$.

In cylindrical coordinates Eq. (1) can be re-written as

$$
\frac{\partial^{2} E}{\partial r^{2}}+\frac{1}{r} \frac{\partial E}{\partial r}+\frac{1}{r^{2}} \frac{\partial^{2} E}{\partial \phi^{2}}-k^{2} E=0 .
$$

Following Kaufman and Keller (1981), the electric field in any layer except the innermost is presented as the sum of incident $\left(E_{\text {inc }}\right)$ and reflected $\left(E_{\text {ref }}\right)$ parts expressed in cylindrical coordinates in terms of a Fourier series (Abramowitz and Stegun, 1972). For layer 1 the incident part is the plane wave, represented in cylindrical coordinates as

$E_{\mathrm{inc}}=E_{0} \exp \left(-k_{1} z\right)=\sum_{0}^{\infty} \alpha_{n} I_{n}\left(k_{1} r\right) \cos n \phi$,

which would exist in an area undisturbed by the cylinder (ambient field in the absence of the cylinder).

The total electric field in the surrounding media (including both ambient plane wave and influence of the cylinder) can be expressed as

$E_{1}=E_{0} \sum_{0}^{\infty} \alpha_{n}\left[I_{n}\left(k_{1} r\right)+R_{1 n} K_{n}\left(k_{1} r\right)\right] \cos n \phi$.

Similarly, for any layer of the cylinder (denoted as $M>m>1$ )

$E_{m}=\sum_{0}^{\infty} A_{m n}\left[I_{n}\left(k_{m} r\right)+R_{m n} K_{n}\left(k_{m} r\right)\right] \cos n \phi$.
In the innermost layer $(M)$, with no reflections

$E_{M}=\sum_{0}^{\infty} A_{M n} I_{n}\left(k_{M} r\right) \cos n \phi$,

where $I_{n}(k r), K_{n}(k r)$ are modified Bessel functions (Abramowitz and Stegun, 1972), $\alpha_{n}=1$ if $n=0, \alpha_{n}=2$ if $n \neq 0, E_{0}$ is the amplitude of undisturbed electric field, $R_{m n}$ are the reflection coefficients at the boundary between layers, $k_{m}$ are the propagation constants in the $m$ layer.

The unknown amplitudes $A_{M n}, A_{m n}$ and reflection coefficients $R_{1 n}, R_{m n}$ can be found from boundary conditions (see Kaufman and Keller, 1981 and also Trichtchenko and Boteler, 2001, for more details).

Electric current in each conductive layer can be derived from the differential form of Ohm's law $j=\sigma E$ as

$I_{m}=\sigma_{m} \int_{0}^{2 \pi} \mathrm{d} \phi \int_{r_{m}}^{r_{m+1}} r E_{m} \mathrm{~d} r$,

where $m$ is the number of the particular conducting layer (except the innermost) with internal radius $r_{m}$ and external radius $r_{m+1}$. Because all harmonics with order $n>0$ will give no current $\left(\int_{0}^{2 \pi} \cos n \phi \mathrm{d} \phi=0\right)$, the final formula for current in any layer except inner is

$$
\begin{aligned}
I_{m}= & \frac{2 \pi \sigma_{m}}{k_{m}} A_{m 0}\left[r_{m+1} I_{1}\left(k_{m} r_{m+1}\right)\right. \\
& -r_{m} I_{1}\left(k_{m} r_{m}\right) R_{m 0}\left(r_{m+1} K_{1}\left(k_{m} r_{m+1}\right)\right. \\
& \left.\left.-r_{m} K_{1}\left(k_{m} r_{m}\right)\right)\right],
\end{aligned}
$$

where $A_{m 0}$ and $R_{m 0}$ are the amplitude and reflection coefficients of the electric field in the layer $m$; and $I_{1}, K_{1}$ are the modified Bessel functions of the first order.

In the innermost layer the electric current is

$I_{M}=\frac{2 \pi \sigma_{M}}{k_{M}} A_{M 0} r_{M-1} I_{1}\left(k_{M} r_{M-1}\right)$.

Similar solutions are valid if the electromagnetic field is produced by a line current. In this case, the electric field in the absence of the cylinder can be expressed as

$E_{\text {inc }}=-\frac{I_{0} i \omega \mu_{1}}{2 \pi} K_{0}\left(k_{1} L\right)$,

where $L$ is the distance from the position of the line current to the point where the field needs to be calculated.

The values of electric fields, currents and effective impedances obtained by using the derived formulas Eqs. (4)(10) are named here the "exact" solution.

It should be noted that in real situations the media is nonuniform due to the existence of the interface between the host medium and overlaid or underlay ones, i.e. due to the contrasts of soil and air or water and air. Because in this paper the emphasis is given to the electric fields and currents inside the cylindrical conductors, the impacts of external conductivity contrasts on electric fields in the media are not modelled. 
Table 1. The electromagnetic parameters and dimensions.

\begin{tabular}{|c|c|c|c|c|c|}
\hline Case & $\begin{array}{c}\# \\
\text { layer }\end{array}$ & $\begin{array}{l}\text { Radius } \\
(\mathrm{m})\end{array}$ & $\begin{array}{r}\text { Conductivity } \\
\left(\sigma, \mathrm{S} \mathrm{m}^{-1}\right)\end{array}$ & $\begin{array}{c}\text { Relative } \\
\text { Permeability } \mu / \\
\text { Permittivity } \varepsilon\end{array}$ & $\begin{array}{c}\text { Media } \\
\text { Conductivity } \\
\left(\sigma, \mathrm{S} \mathrm{m}^{-1}\right) \\
\text { Relative } \\
\text { Permittivity } \varepsilon\end{array}$ \\
\hline \multirow[t]{2}{*}{ Cable } & 1 & 0.002 & $10^{-15}$ & $\varepsilon=2.3$ & soil \\
\hline & 2 & 0.0016 & $5.8 \times 10^{+7}$ & $\mu=1$ & $\begin{array}{cc}\sigma=10^{-3} & \sigma=1 \\
\varepsilon=1 & \varepsilon=1\end{array}$ \\
\hline Transmission & 1 & 0.012 & $3.5 \times 10^{+7}$ & $\mu=1$ & \multirow[t]{2}{*}{$\sigma=10^{-14}$} \\
\hline line & 2 & 0.0024 & $10^{+7}$ & $\mu=1$ & \\
\hline \multirow[t]{3}{*}{ Pipeline } & 1 & 0.501 & $10^{-6}$ & $\varepsilon=5$ & \multirow[t]{3}{*}{$\sigma=10^{-3}$} \\
\hline & 2 & 0.5 & $10^{+7}$ & $\mu=250$ or 1 & \\
\hline & 3 & 0.49 & $10^{-6}$ & $\varepsilon=2$ & \\
\hline Rail & 1 & 0.0501 & $4.4 \times 10^{+6}$ & $\mu=1$ & $\sigma=10^{-14}$ \\
\hline
\end{tabular}

\section{Input parameters}

The following samples of the conducting structures were used: typical rail insulated from the ground, insulated solid copper wire used in many applications, bare aluminium conductor steel reinforced (ACSR) used in overhead transmission lines, and coated steel pipeline with gas inside. For the external media air, soil and water are considered where applicable.

The electromagnetic characteristics and the dimensions of the conductive layered structures and the external media are presented in Table 1, with values taken from the references described below.

Diameters of the copper wires and cables vary widely, from $1.6 \mathrm{~mm}$ (protected ground wire) to $4.01 \mathrm{~mm}$ (telecommunication power cables, leads for transformers, solenoids, etc.,) to $18 \mathrm{~mm}$ (wires for heating equipment) which can be found in multiple on-line catalogues, for example in http://www.southwire.com/products/ProductCatalog. htm or in Technical Information Handbook, Wire and Cables (2013). The mid-range parameters of a single insulated copper wire have been chosen with the outer radius (with the polyethylene insulation) of $2 \mathrm{~mm}$ and inner radius of $1.6 \mathrm{~mm}$. The characteristics of the insulation were taken from Warne (2005).

Transmission line ACSR overhead conductor diameters also have a range of values from 1.7 to $12.7 \mathrm{~mm}$ for steel core (single) and from 5 to $39 \mathrm{~mm}$ for the whole wire. The wire with inner steel reinforcement radius of $2.4 \mathrm{~mm}$ and the outer radius of aluminium conductor of $12 \mathrm{~mm}$ (corresponding to the "Kingbird" from the above-cited catalogue) has been used in this study.
The pipeline parameters were adopted from Peabody (2001) and are the same as in Trichtchenko and Boteler (2001) with inner radius of steel equal to $0.49 \mathrm{~m}$, outer radius of steel of $0.5 \mathrm{~m}$ and coating thickness of $1 \mathrm{~mm}$. The magnetic properties of the pipeline steels are highly variable, thus, two cases of the relative magnetic permeabilities were chosen, $\mu=1$ and $\mu=250$ (Dawalibi and Southey, 1990).

The rail electromagnetic parameters and dimensions are taken from Mariscotti and Pozzobon (2004). In order to solve the problem analytically, the shape of the rail cross section is replaced by a solid cylinder with the radius of $48.4 \mathrm{~mm}$ that has the same cross-sectional area as the cross-sectional area of the rail.

Electrical conductivity of the host media has been chosen to range from resistive earth $\left(10^{-3} \mathrm{~S} \mathrm{~m}^{-1}\right)$ to conductive water $\left(1 \mathrm{~S} \mathrm{~m}^{-1}\right)$ (see, for example, Simpson and Bahr, 2005) with the permittivity of free space $\left(4 \pi \times 10^{-7}\right)$; the air conductivity used $\left(10^{-14} \mathrm{~S} \mathrm{~m}^{-1}\right)$ corresponds to its value given in Haynes (2015).

The properties and dimensions for all discussed layered structures and media are presented in Table 1. It should be noted that values presented in Table 1 serve as representative of a wide range of examples to be used as inputs into models discussed in the paper. In principle, these input values can be changed based on particular specifications of some particular case.

\section{Illustrative example for a single frequency}

To better understand the general results obtained for multiple frequencies presented further in Sect. 5, the detailed view of 

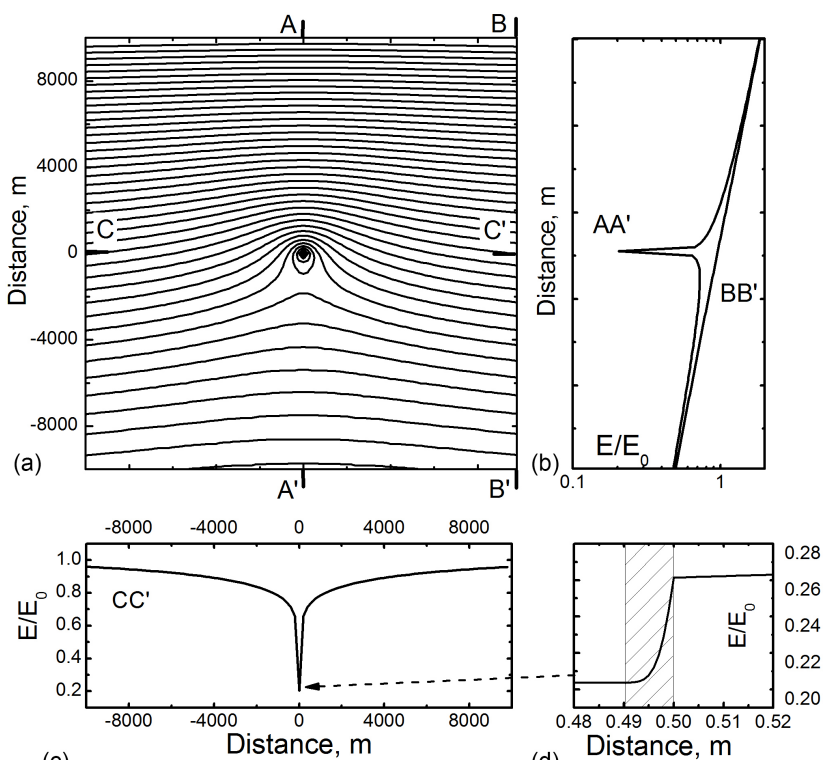

(c)

(d)

Figure 2. The topology of the normalized absolute value of the electric field for the frequency of $1 \mathrm{~Hz}$, conductivity of the external media is $10^{-3} \mathrm{~S} \mathrm{~m}^{-1}$, disturbed by the multilayered pipeline with the relative permeability of the steel being 250 and other parameters as in Table 1. (a) contour lines; (b) values along the cross sections at $X \sim 0 \mathrm{~m}$ (AA') and $X \sim 10 \mathrm{~km}$ (BB'); (c) values along cross section at $Z=0 \mathrm{~m}\left(\mathrm{CC}^{\prime}\right)$ and (d) magnified view across the pipeline wall, see more detailed explanations in the text.

interaction of the plane electromagnetic wave with multilayered infinitely long cylinder at a particular single frequency is first discussed below. To represent the most complex structure from those listed in Table 1, the case of the pipeline with permeable steel $(\mu=250)$, embedded in the soil has been chosen. It is comprised of three layers: insulator, steel and gas. In this example the incident plane wave has a frequency of $1 \mathrm{~Hz}$. Electromagnetic parameters of all components are as listed in the Table 1.

The set of results is presented in Fig. 2, which consists of four sub-figures. Fig. 2a shows the contour lines of the absolute value of normalized electric field $E / E_{0}$, where $E_{0}$ is the electric field in the absence of the conductive structure at the location of the centre of the cylinder with coordinates $(0.0,0.0)$. Figure $2 \mathrm{a}$ covers the area of $10 \times 10 \mathrm{~km}^{2}$. Letters AA', BB' and CC' at the periphery of the Fig. 2a denote the locations of the cross-sections which are plotted in Fig. $2 b$ for two vertical cross sections AA' and BB', and in Fig. 2c for horizontal cross-section CC'. Figure $2 d$ presents the close view through the pipeline steel, covering only distances from 0.48 to $0.52 \mathrm{~m}$.

Changes from the plane wave geometry to the circular geometry of the electric field is clearly seen in Fig. 2a and, as expected, at the surface of the cylindrical structure the wave is becoming circular. The changes of the amplitude of the electric field downwards are demonstrated in Fig. 2b. At the distance of $10 \mathrm{~km}$ from the cylinder (cross-section BB') the fall-off of the plane wave is defined by the exponential attenuation in the media (soil) with no influence by the conductor (see Eq. 4). The cross-section through the cylinder (AA') clearly demonstrates the additional attenuation produced by the conductive structure. Thus, it is easy to see that the electric field at the surface of the conductive structure is not the same as its ambient value, at least at this particular frequency. The horizontal cross-section CC' (Fig. 2c) shows the same attenuation in electric field due to conductive structure, confirming the result.

Details of the electric field behaviour inside the conductive structure are presented in Fig. 2d, where the shaded area is the pipeline steel layer (coordinate range is from 0.49 to $0.50 \mathrm{~m}$ ), demonstrate the frequency-dependent skin-effect. Thus, modelling of the conductive structures as purely DCresistances definitely has its limitation and can hardly be applicable to the case illustrated above, which motivates further investigations.

\section{Frequency-dependent results}

The formulas derived on the basis of the analytical solutions shown above were applied to estimate the frequency dependence of the variations of electromagnetic field and currents at any location of the single conductor or multi-layered cylinder and internal media. In this section the results of calculations of the electric field at two surfaces of the same conductive layer in four types of conductive multilayered structures for the continuous spectrum of the electromagnetic field variations are presented. The frequency dependencies are shown in Figs. 3-6, where the outermost surface of each conductive layer is denoted as "exterior" and the inner surface as "interior", or "close to the centre" for the innermost conductors.

The simplest of the four cases would be the bare steel rail surrounded by air (Fig. 3). The solid lines represent the falloff of the electric field (relative to the ambient field) in terms of amplitude (top plot) and phase (bottom plot) at the external surface of the steel, and dotted lines - close to the centre of the steel cylinder. Our approach cannot produce the analytical solution for the true shape of the rail cross-section, thus, a solid cylinder with the same cross-sectional area has been used for calculations. For the frequencies of $0.1 \mathrm{mHz}$ to $0.1 \mathrm{~Hz}$ (corresponding to periods of geomagnetic variations, such as the $1 \mathrm{~h}, 15 \mathrm{~min}, 1 \mathrm{~min}$ ) there is practically no influence of the conductor and, therefore, for geomagnetic interference modelling the ambient electric field and DC resistance can be used. For frequencies from 0.1 to $1 \mathrm{~Hz}$, there are changes up to $45^{\circ}$ in phase, but insignificant changes in amplitude, while for frequencies above $1 \mathrm{~Hz}$ the ambient electric field approximation might introduce the errors, and the changes in phase between the surface and centre of the cylinder are seen. From about $30 \mathrm{~Hz}$ the difference between the amplitudes of the field at the exterior surface and centre of 

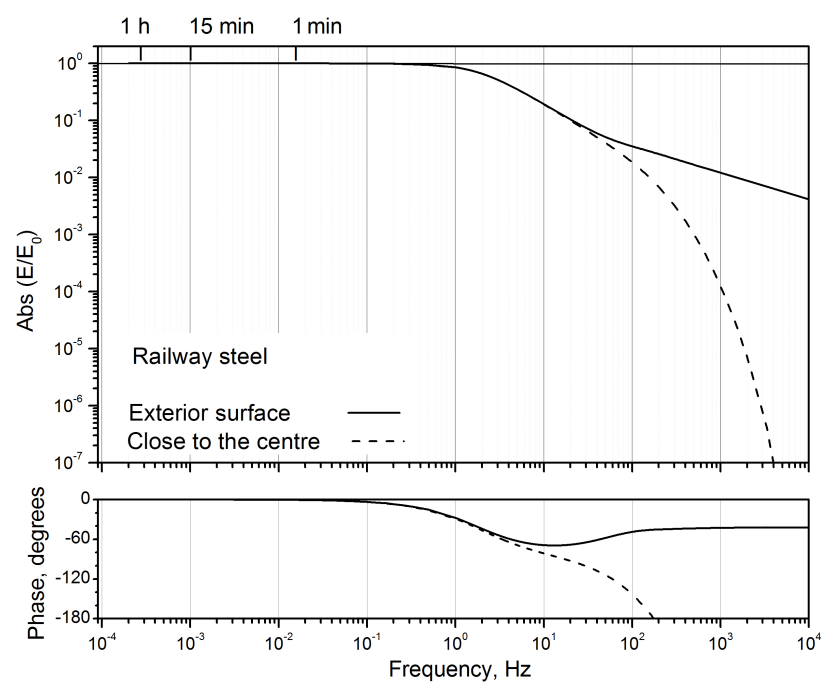

Figure 3. The frequency dependencies of normalized absolute amplitude (top) and phase (bottom) of the electric field at the outer surface and near the centre of the railway steel. External media is air with conductivity of $10^{-14} \mathrm{~S} \mathrm{~m}^{-1}$.

the rail are clear, thus the approximation of DC resistance should be avoided, or at least acknowledged as the source of possible error.

In the case of the insulated copper wire (Fig. 4), the absolute value of the normalised electric field in the conductor is the same as for the ambient electric field and is independent of frequency up to approximately $30 \mathrm{~Hz}$, but for the phase only up to $10 \mathrm{~Hz}$. The electric field at the outer and inner surfaces is approximately the same at frequencies up to $2 \mathrm{kHz}$, while the skin effect on deviations of the phases at these two surfaces has a visible effect at frequencies of $0.3 \mathrm{kHz}$. The influence of the conductivity of the external media is in making the deviations from the ambient field smaller when there is less contrast between the media and conductor conductivities (i.e. less for the sea-copper scenario than for soil copper).

In the case of the large multilayered structure such as a pipeline (Fig. 5), the electric field at the surface of the steel layer can be regarded as the ambient field only up to $\sim 0.017 \mathrm{~Hz}$ (period of $1 \mathrm{~min}$ ). For two cases of magnetic permeabilities, the skin effect is significant at lower frequencies for higher permeable steel $(\mu=250)$ and is seen in changes of phases at exterior and interior surfaces at $0.1 \mathrm{~Hz}$. For steel with $\mu=1$ the skin effect is appearing at the frequency of $10 \mathrm{~Hz}$.

Changes of the electric field with frequency for the case of a cylinder consisting of the two conducting layers without insulator (transmission line wire comprised of aluminium with a steel core) are presented in Fig. 6. The electric field has been calculated for four different locations: exterior surface of aluminium (black solid line), interior surface of aluminium (black dashed line), near the exterior surface of steel (dashed red line) and close to the centre of the steel (blue

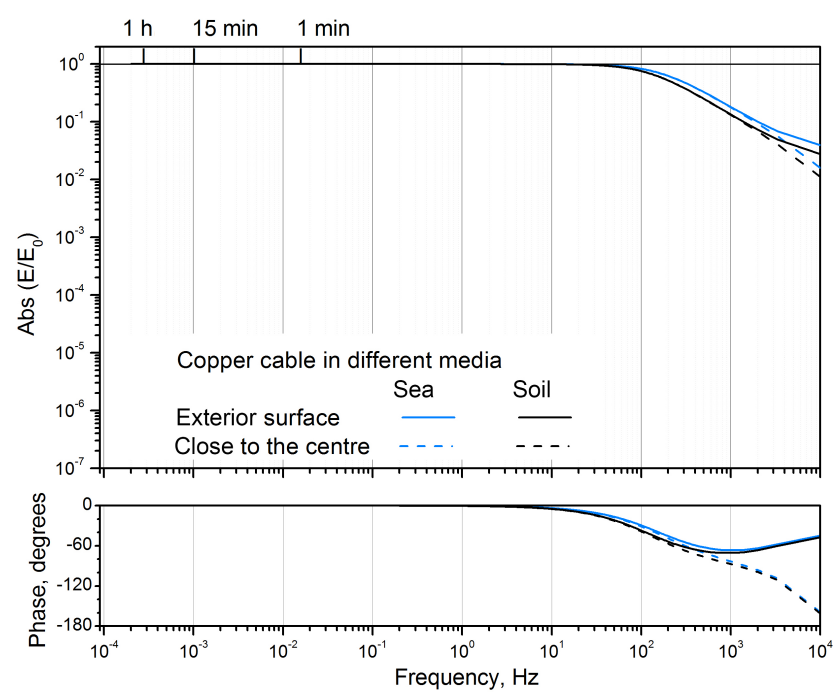

Figure 4. The frequency dependencies of normalized absolute amplitude (top) and phase (bottom) of the electric field at the outer surface and near the centre of the copper layer in cable, for two cases of external media, soil with conductivity of $10^{-3} \mathrm{~S} \mathrm{~m}^{-1}$ and sea with conductivity of $1 \mathrm{~S} \mathrm{~m}^{-1}$.

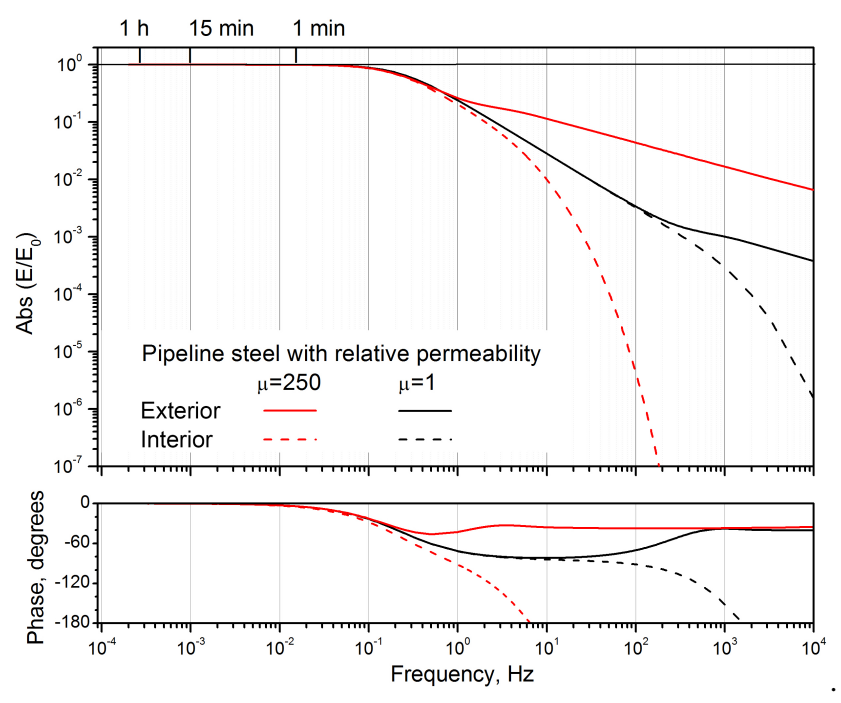

Figure 5. The frequency dependencies of normalized absolute amplitude (top) and phase (bottom) of the electric field at the outer surface and near the centre of the steel in pipeline with relative permeabilities of 1 (black lines) and 250 (red lines), embedded in soil with conductivity $10^{-3} \mathrm{~S} \mathrm{~m}^{-1}$

dashed line). As is expected from the boundary conditions, the electric field at the interior surface of aluminium is the same as the electric field at the surface of the steel. There is no noticeable drop-off in the electric field of the steel for the frequencies shown in Fig. 6.

As can be inferred from the presented results, the electric field in transmission line can be approximated by the ambient field up to frequencies of $0.1 \mathrm{~Hz}$. In case of not taking 


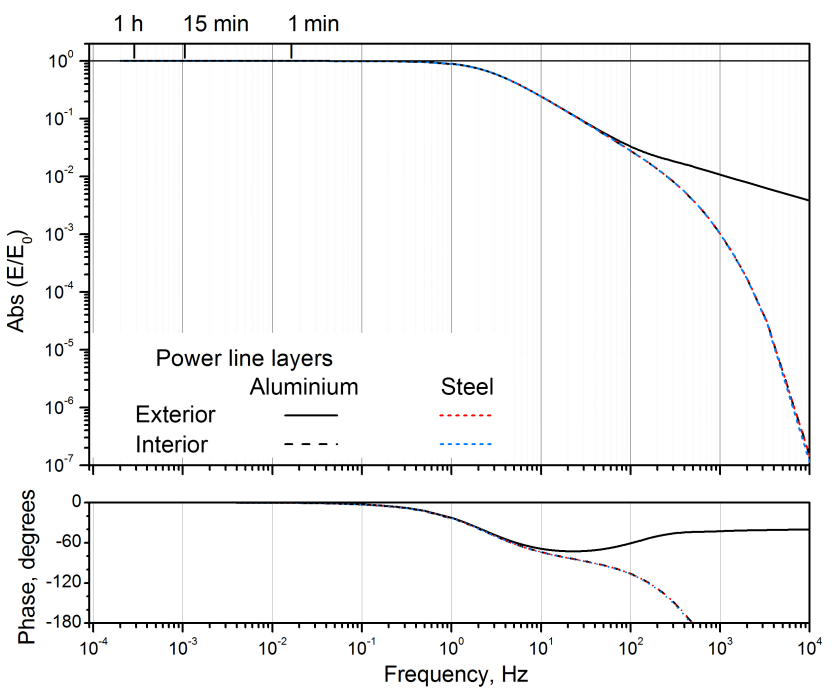

Figure 6. The frequency dependencies of normalized absolute amplitude (top) and phase (bottom) of the electric field at the outer and inner surfaces of the aluminium (black lines) and steel (red and blue lines) in transmission line wire, located in air with conductivity $10^{-14} \mathrm{~S} \mathrm{~m}^{-1}$.

into account the phase, i.e. if there are no concerns about the time delays, but only with the amplitude of the electric field in time domain, then ambient field approximation can be extended to close to $1 \mathrm{~Hz}$. The skin effect in the aluminium is not significant until $100 \mathrm{~Hz}$ for amplitude, but is clearly seen in phase starting at about $10 \mathrm{~Hz}$.

\section{Evaluating the effective impedance}

One of the most important characteristics for practical applications is the ratio of the electric field in the absence of the conductive structure (which can be found from geophysical modelling or magnetotelluric measurements) to the total current in the conductor (i.e. the GIC). The term "effective impedance" is used here for this ratio.

$Z_{\text {eff }}=E_{0} / I$

Thus, in order to find the current in the conductive layer, one needs to divide the ambient electric field (i.e. independent on the conductive structure) at the location of the conductor by the effective impedance. The exact value of this effective impedance is obtained using derived Eqs. (9) and (10) and is compared with the approximate formulas presented below.

In order to obtain approximate formula, the effective impedance is defined as the sum of two parts. One part is influenced by the external media and can represent the dissipation of the energy outside of the conductor (as, for example, in Dawalibi and Southey, 1989), i.e. defined by the properties of media (layer 1), and another is taking into account the properties of the conductive cylinder and skin-effect, i.e. defined by the properties of the conductor.

$Z_{\text {eff }}=\left(Z_{1}+Z_{\text {cond }}\right)$

The $Z_{1}$ term has been approximated as the ratio of the electric field produced by the infinitely thin current line $\left(I_{\text {line }}\right)$ at the distance of the external radius of the conductor $\left(r_{1}\right)$ to the value of this current. It does not depend on the value of the current and can be expressed as (see also Eq. 11)

$Z_{1}=\frac{E_{1}}{I_{\text {line }}}=-\frac{i \omega \mu_{1}}{2 \pi} K_{0}\left(k_{1} r_{1}\right)$

After approximation for small arguments, which is valid in the low-conductive media (in contrast to the conductive network), the resulting expression is

$Z_{1}=\frac{i \omega \mu_{1}}{2 \pi}\left(\ln \frac{2}{k_{1} r_{1}}-0.5772\right)$,

where $\mu_{1}$ and $k_{1}$ are magnetic permeability of the external media $\left(\mu_{1}=\mu_{0}\right.$, i.e. the free space value) and propagation constant of external media, $r_{1}$ is the external radius of conductor (Fig. 1) and $\ln$ is the natural logarithm.

Impedance of conductor $\left(Z_{\text {cond }}\right)$ is approximated as in Sunde (1968), for the case of large propagation constant of the conducting layer $m$.

$Z_{\text {cond }}=R_{\mathrm{dc}} k_{m}\left(r_{m}-r_{m+1}\right) \operatorname{coth}\left(k_{m}\left(r_{m}-r_{m+1}\right)\right)$,

where $R_{\mathrm{dc}}$ is the DC resistance per unit length, $k_{m}$ is the propagation constant, $r_{m}-r_{m+1}$ is the thickness of the conductive layer and coth is the hyperbolic cotangent.

The approximate estimation of the total impedance as the sum of Eqs. (15) and (16) has been compared with the effective impedance calculated as the ratio of the ambient electric $E_{0}$ to the total current defined by Eqs. (11) or (12). Results for the cases of rail, copper cable in two media, steel pipe with two magnetic permeabilities and the transmission line are presented in Figs. 7-10. The results corresponding to the approximate formula are presented only for several frequencies for the sake of clarity of the plots.

Frequency dependence of the impedances of the steel rail is presented in Fig. 7. The real part of the impedance is constant for frequencies up to $\sim 2 \mathrm{~Hz}$, at higher frequencies the skin effect comes into account and the real part became frequency-dependent. The imaginary part at frequency of $\sim 0.3 \mathrm{~Hz}$ is approximately 1 order of magnitude smaller than real part, thus until this frequency the imaginary part can be neglected and the impedance can be regarded as the DC resistance. The coincidence of the approximate formula and exact formula is clear.

Frequency dependence of the impedances of copper cable for two different external media (blue for sea, black for soil) are presented in Fig. 8. The real parts of the impedances are the same in both cases of media, either for the exact (solid lines overlap) or approximate (stars and open squares overlap). The slight deviations between results from exact and 


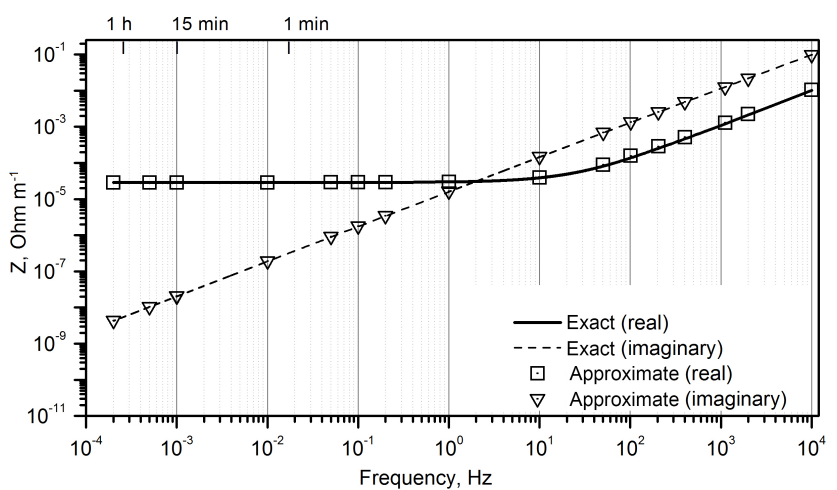

Figure 7. The frequency dependence of impedance of the steel rail calculated by use of the exact (lines) and approximate methods (scatter symbols).

approximate formula are only marginal at the highest frequency of our study (i.e. at $10 \mathrm{kHz}$ ). The imaginary parts of impedance in two media are slightly different, larger in the case where the contrast between the media and conductor conductivities are larger. Approximate and exact formulas give the same results for the discussed frequency range.

The DC approximation for the cable network model can be used up to $10 \mathrm{~Hz}$, i.e. until the imaginary part is an order of magnitude less. For frequencies above $\sim 1 \mathrm{kHz}$ the real part increases with frequency (skin effect) and the imaginary part is an order of magnitude larger.

Variations of impedances of steel pipeline for two different relative magnetic permeabilities with frequency are presented in Fig. 9. As in the two previous cases, the derived impedance and approximate impedance give practically the same results in the discussed frequency range. Frequency at which the DC approximation can be used (i.e. the real part is an order of magnitude larger than imaginary) is very close to $0.017 \mathrm{~Hz}$ (period of $1 \mathrm{~min}$ ). The relative magnetic permeability of 250 is causing the real part of impedance to increase only marginally faster in the frequency range of $1 \mathrm{~Hz}$ to $1 \mathrm{kHz}$, than for the magnetic permeability of free space. This deviation is due to the extra induction.

For the case of a transmission line, when the two conductive layers (aluminium and steel) are in parallel, the impedance of the conductive layer is

$Z_{\text {cond }}=Z_{\mathrm{Al}} \times Z_{\text {steel }} /\left(Z_{\mathrm{Al}}+Z_{\text {steel }}\right)$.

The results are presented in Fig. 10, with the real part (solid line) being constant for low frequencies (up to $3 \mathrm{~Hz}$ ), and the imaginary part (dashed line) being about an order of magnitude smaller for frequencies below $0.2 \mathrm{~Hz}$. For frequencies above $3 \mathrm{~Hz}$ the real part increases with frequency (skin effect) and the imaginary part is also increasing and became an order of magnitude larger.

The approximate expression of impedance gives exactly the same values and is represented as open squares (real part) and open triangles (imaginary part).

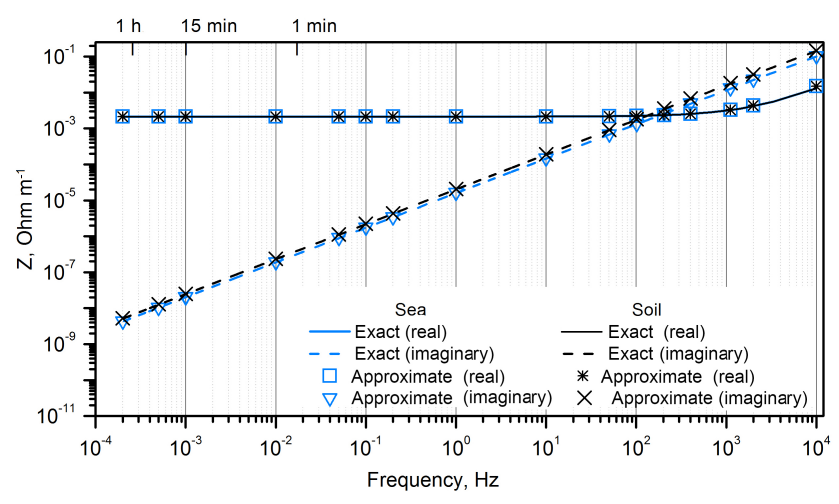

Figure 8. The frequency dependence of impedance of the copper cable in sea water with conductivity of $1 \mathrm{~S} \mathrm{~m}^{-1}$ and in soil with conductivity of $10^{-3} \mathrm{~S} \mathrm{~m}^{-1}$, calculated by the exact (lines) and approximate (scatter) methods.

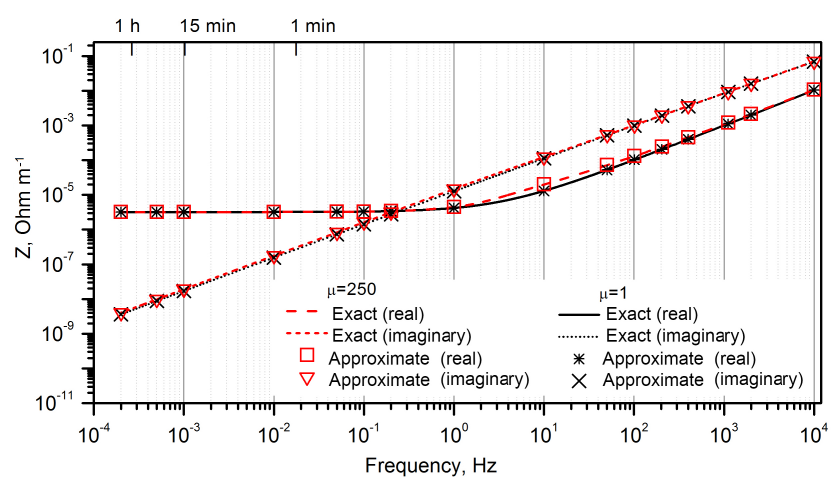

Figure 9. The frequency dependence of impedance of the steel in pipeline with relative permeabilities of 1 and 250 and external media with conductivity $10^{-3} \mathrm{~S} \mathrm{~m}^{-1}$ calculated by the exact (lines) and approximate (scatter) methods.

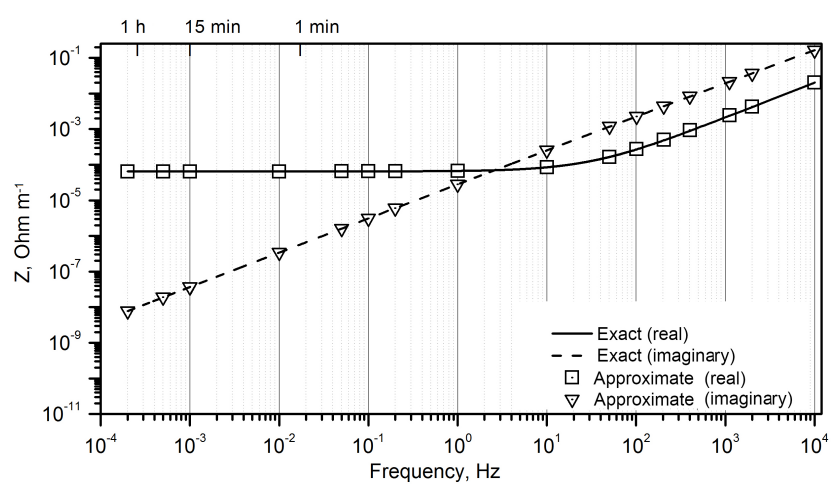

Figure 10. The frequency dependence of impedance of the transmission line wire, for external media with conductivity $10^{-14} \mathrm{~S} \mathrm{~m}^{-1}$ calculated by exact (lines) and approximate (scatter) methods. 


\section{Conclusions}

The presented analytical solution of an idealized case of electromagnetic field interaction with an infinitely long multilayered cylindrical conductor has provided several useful results. The analytical solution can be applied over a wide range of scales, from inside a millimetre-size wire to the kilometres of distances into the media and for a wide range of frequencies, from $\mathrm{mHz}$ to $\mathrm{kHz}$.

The resolution to which the model allows to calculate the electromagnetic field is very high, so that slight changes inside a small structure (centimetres) can be resolved as well as 10 orders of magnitude changes and kilometres away into the surrounding media, as demonstrated in Sect. 3.

Several results of practical importance were obtained. It has been shown that the driving electric field inside the conductor cannot be regarded as the ambient field in general modelling, but the approximation is valid for low frequencies (Sect. 4). It also has been shown that the conductivity of the surrounding media and the magnetic permeability of the conductive layer change the behaviour of the electric field inside the conductive layer.

Calculations of the effective impedance (Sect. 5) show that for low frequencies (less than some particular frequency) the real part of the effective impedance is constant and larger than the imaginary part, while for higher frequencies this assumption can lead to large errors. Thus, for frequencies above that particular frequency (different for each particular case of conductive structure) when applying, for example, transmission line network modelling for calculations of geomagnetically induced currents, the impedances of wires needs to be of a complex value.

The developed exact analytical solution enables testing of the approximate formula adopted from the literature. Verification of the effective impedances calculated from approximations and using expressions obtained in this paper show their practically perfect coincidence. Therefore, the applicability of the total impedance as sum of Eqs. (15) and (16) can be extended to the frequencies from $0.1 \mathrm{mHz}$ to $10 \mathrm{kHz}$ for the electromagnetic parameters considered in this study (see Table 1). This interval covers the complete range of geomagnetic variations of geophysical nature, such as sudden impulses and/or sudden commencements, pulsations and substorm and/or storm - related fast variations. Therefore, they can be recommended for use in the space weather applications when the fast variations of the geomagnetic fields are of specific concern, such as for power grids, railways and perhaps other applications. Several possible continuations of the work are foreseen, one is to properly numerically estimate the impact of surface conductivity contrast (air-soil and soilwater). Also, the comparisons and validations could be done by application of the DC and frequency-dependent models for particular case studies when the recordings of GIC at frequencies higher than $1 \mathrm{~Hz}$ are available.
The resulting exact solutions can also serve as a benchmark for numerical models or provide additional insight into the application of some known approximate methods and formulas.

Acknowledgements. The author gratefully acknowledges discussions with David Boteler and Risto Pirjola.

The topical editor, G. Balasis, thanks two anonymous referees for help in evaluating this paper.

\section{References}

Abramowitz, M. and Stegun, I. A.: Handbook of Mathematical Functions with Formulas, Graphs, and Mathematical Tables, 9th printing, Dover, New York, USA, 1972.

Boteler, D. H., Pirjola, R. J. and Nevanlinna, H.: The effects of geomagnetic disturbances on electrical systems at the Earth's surface, Adv. Space Res., 22, 17-27, 1998.

Campbell, W. H.: Induction of auroral zone electric currents within the Alaska pipeline, Pure Appl. Geophys., 116, 1143-1173, 1978.

Campbell, W. H.: Introduction to Geomagnetic Fields, Cambridge University Press, Cambridge, UK, 1997.

Dawalibi, F. P. and Southey, R. D.: Analysis of electrical interference from power lines to gas pipelines. Part I: Computation methods, IEEE T. Power Deliver, 4, 1840-1846, 1989.

Dawalibi, F. P. and Southey, R. D.: Analysis of electrical interference from power lines to gas pipelines. Part II: Parametric Analysis, IEEE T. Power Deliver, 5, 415-421, 1990.

Gummow, R.: GIC effects on pipeline corrosion and corrosion control systems, J. Atmos. Sol.-Terr. Phy., 64, 1755-1764, 2002.

Haynes, W. M.: Handbook of Chemistry and Physics, 96th edition, CRC Press, Boca Raton, FL, USA, 2015.

Intermagnet website: http://intermagnet.org/publication-software/ technicalsoft-eng.php, last access: 22 March 2016.

Kappenman, J.: An overview of the impulsive geomagnetic field disturbances and power grid impacts associated with the violent Sun-Earth connection events of 29-31 October 2003 and a comparative evaluation with other contemporary storms, Adv. Space Res., 3, S08C01, doi:10.1029/2004SW000128, 2005.

Kaufman, A. A. and Keller, G. V.: The magnetotelluric sounding method, in: Methods in geochemistry and geophysics, Elsevier, Amsterdam, the Netherlands, 15, 187-192, 1981.

Lanzerotti, L. J. and Gregori, G. P.: Telluric currents: The natural environment and interaction with man-made systems, in: The Earth's electrical environment, National Academy Press, Washington, DC, USA, 232-257, 1986.

Mariscotti, A. and Pozzobon, P.: Determination of the electrical parameters of railway traction lines: calculation, measurement and reference data, IEEE T. Power Deliver., 19, 1538-1546, 2004.

On-line catalogues of wires: http://www.southwire.com/products/ ProductCatalog.htm, last access: 22 March 2016.

Peabody, A. W.: Control of Pipeline corrosion, 2nd edition, NACE International, Houston, TX, USA, ISBN 1-57590-092-0, 2001.

Pirjola, R.: Review on the calculation of surface electric and magnetic fields and of geomagnetically induced currents in groundbased technological systems, Surv. Geophys., 23, 71-90, 2002. 
Ptitsyna, N. G., Kasinskii, V. V., Villoresi, G., Lyahov, N. N., Dorman, L. I., and Iucci, N.: Geomagnetic effects on mid-latitude railways: A statistical study of anomalies in the operation of signaling and train control equipment on the East-Siberian Railway, Adv. Space Res., 42, 1510-1514, 2008.

Simpson, F. and Bahr, K.: Practical Magnetotellurics, Cambridge University Press, Cambridge, UK, 2005.

Sunde, E. D.: Earth conduction effects in transmission system, Dover, New York, USA, 1968.

Technical Information Handbook: Wire and Cables, Copyright Anixter, ISBN: 978-0-615-24926-1, 2310 Patriot Blvd, IL, USA, 60026, 5th edition, 2013.
Trichtchenko, L. and Boteler, D. H.: Specification of geomagnetically induced electric fields and currents in pipelines, J. Geophys. Res., 106, 21039-21048, 2001.

Viljanen, A.: Geomagnetically Induced Currents in the Finnish Natural Gas Pipeline, Geophysica, 25, 135-159, 1989.

Warne, D. F.: Newnes Electrical Power Engineer's Handbook, The Newnes, imprint of Elsevier, Oxford, UK, 2005

Wik, M., Pirjola, R., Lundstedt, H., Viljanen, A., Wintoft, P., and Pulkkinen, A.: Space weather events in July 1982 and October 2003 and the effects of geomagnetically induced currents on Swedish technical systems, Ann. Geophys., 27, 1775-1787, doi:10.5194/angeo-27-1775-2009, 2009. 